\title{
Contrast response functions for single Gabor patches: ROI-based analysis over-represents low-contrast patches for GE BOLD
}

\author{
Jennifer F. Schumacher ${ }^{1+}$, Serena K. Thompson ${ }^{1}$, Cheryl A. Olman ${ }^{1,2,3^{*}}$ \\ Graduate Program in Neuroscience, University of Minnesota, Minneapolis, MN, USA \\ 2 Department of Psychology, University of Minnesota, Minneapolis, MN, USA \\ ${ }^{3}$ Department of Radiology, University of Minnesota, Minneapolis, MN, USA
}

Edited by:

Roland G. Henry, University of

California at San Francisco, USA

Reviewed by:

Srikantan S. Nagarajan, University of

California at San Francisco, USA

Handwerker A. Daniel, National

Institutes of Health, USA

Kornak John, University of California,

USA

\section{*Correspondence:}

Cheryl A. Olman, Department of

Psychology, University of Minnesota,

N218 Elliott Hall, 75 East River Road,

Minneapolis, MN 55455, USA.

e-mail:caolman@umn.edu

${ }^{+}$Present address:

Jennifer F. Schumacher, 3M Company,

St Paul, MN, USA
Important for the interpretation of BOLD fMRI data is a linear relationship between the BOLD response and the underlying neural activity: increased BOLD responses should reflect proportionate increases in the underlying neural activity. While previous studies have demonstrated a linear relationship between the peak amplitude of the BOLD response and neural activity in primary visual cortex (V1), these studies have used stimuli that excite large areas of cortex, and the linearity of the BOLD response has not been demonstrated when only a small patch of cortex is stimulated. The BOLD response to isolated Gabor patches of increasing contrast was measured with gradient echo (GE) BOLD and spin echo (SE) BOLD at 7T. Our primary finding is notable spatial heterogeneity of the BOLD contrast response, particularly for the GE BOLD data, resulting in a more reliably linear relationship between BOLD data and estimated neural responses in the center of the cortical representations of the individual Gabor patches than near the edges. A control experiment with larger sinusoidal grating patches confirms that the observed sensitivity to voxel selection in the regions of interest-based analysis is unique to the small stimuli.

Keywords: fMRI, primary visual cortex, spin echo BOLD, contrast, psychophysics

\section{INTRODUCTION}

Many studies have investigated the linearity of the relationship between BOLD signal changes and measured or inferred neural activity and have found that neurohemodynamic coupling behaves, by and large, as a linear system that obeys the principles of superposition and scaling (Boynton et al., 1996, 1999; Logothetis et al., 2001; Heckman et al., 2007; Zhang et al., 2008). Because these previous studies have used full-field or extended visual stimuli, which stimulate large regions of cortex, the present study was designed to test the linearity of the BOLD response to isolated Gabor patches that produce well-localized patches of neural activity (approximately $5 \mathrm{~mm}$ in diameter) in early visual areas. These relatively weak stimuli could potentially unveil non-linear aspects of the hemodynamic response, such as local oxygen consumption without concomitant increases in local blood flow (resulting in a lower-than-predicted BOLD response to low-contrast stimuli), or over-perfusion at low contrast due to a lack of fine resolution in local blood flow recruitment. The expected finding for this experiment is a linear relationship when the BOLD contrast response is compared against the neural contrast response estimated from psychophysical data.

As an alternative to the more common gradient echo (GE) BOLD techniques that use $\mathrm{T}_{2}{ }^{*}$-weighted images to maximize sensitivity to magnetic field perturbations in and near veins (Ogawa et al., 1993; Bandettini et al., 1994), spin echo (SE) BOLD techniques use $\mathrm{T}_{2}$-weighted images to bias BOLD sensitivity toward small venules and capillaries (Norris et al., 2002; Duong et al., 2003;
Yacoub et al., 2003; Hulvershorn et al., 2005; Parkes et al., 2005). At low field strengths (1.5 T), the spatial specificity of SE BOLD is limited by intravascular blood signal from large veins (Oja et al., 1999), but at high field strength (e.g., $7 \mathrm{~T}$ ), the short $\mathrm{T}_{2}$ of venous blood removes this residual contribution from large veins (Duong et al., 2003). We therefore measured the BOLD contrast response with both GE and SE techniques at $7 \mathrm{~T}$ to discover whether any non-linearities in the BOLD response are more likely attributable to large-diameter or small-diameter vascular compartments.

\section{MATERIALS AND METHODS SUBJECTS}

Functional MRI data were collected from eight human subjects (five female, ages 25-35) with normal or corrected to normal vision. Subjects participated in one or both of the experiments described below after providing written informed consent. The experimental protocols conformed to safety guidelines for MRI research and were approved by the Institutional Review Board at the University of Minnesota.

\section{VISUAL STIMULI}

The visual stimuli (Figure 1) consisted of four Gabor patches, one located in each of the four visual quadrants at $3^{\circ}$ eccentricity. Each Gabor patch consisted of a 3 cycles-per-degree (cpd) sinusoidal grating modulated by a Gaussian envelope with full width at halfmaximum of $0.6^{\circ}\left(\sigma=0.25^{\circ}\right)$. All stimuli were presented on a mean gray background. Stimuli were generated and presented with Matlab 


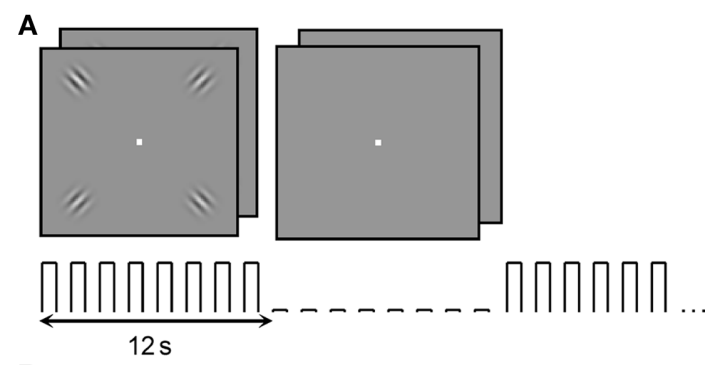

B

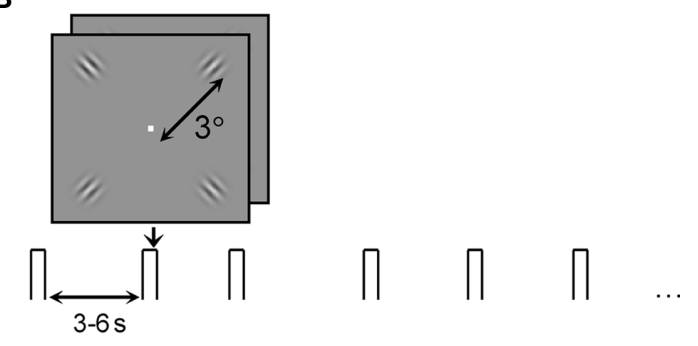

FIGURE 1 | Stimuli used to study the BOLD fMRI contrast response in V1. (A) Block-design localizer scans were used to select regions of interest for analysis of the event-related data. (B) Stimuli for event-related scans were presented in a 2 IFC paradigm at $5,10,30$, and $90 \%$ contrast with inter-trial intervals randomly distributed between 3 and $6 \mathrm{~s}$.

(R2007b; Mathworks, Inc., Natick, MA, USA) using the Psychtoolbox extensions (Brainard, 1997; Pelli, 1997). Macintosh iMac computers with $2.4 \mathrm{GHz}$ Intel Core 2 Duo processors (4 GB RAM) running OS 10.5 .7 served as the processors for the psychophysics and fMRI systems. For psychophysical measurements of contrast discrimination thresholds, stimuli were displayed on a NEC 2180UX LCD monitor, subtending $8^{\circ} \times 11^{\circ}$ of visual angle at a viewing distance of $200 \mathrm{~cm}$. For the $7 \mathrm{~T}$ experiments, stimuli were projected onto a screen behind the subjects' heads (Sanyo projector with custom lens made by Navitar), which was viewed via a mirror mounted on the head coil. Total image area subtended $12^{\circ} \times 16^{\circ}$ at a viewing distance of $71 \mathrm{~cm}$.

For the psychophysics computer, a linear luminance response across the entire dynamic range was ensured by measuring the brightness of the screen (using a Minolta CS-100, Minolta Corporation, USA) at 64 points uniformly distributed across the monitor's dynamic range and calculating a look-up table that would produce a linear luminance response. Because of the high field environment, direct measurement of the screen luminance for the fMRI experiment was not possible. We therefore used a variant of the VisualGamma calibration routine provided with version 3 of Psychtoolbox to ensure a linear brightness response on the projector screen. This procedure divides the dynamic range of the monitor into eight sections and uses a human observer to estimate the look-up table value that produces a brightness midway between the maximum and minimum luminance of each segment. While this procedure produces a reliable linear look-up table, it provides no absolute information about screen luminance, so the contrast values reported in this paper refer to contrast calculated in the image rather than luminance contrast.

For the control experiment, larger patches of $3 \mathrm{cpd}$ sinusoidal gratings replaced the Gabors. The patches were centered at $3^{\circ}$ eccentricity and had a radius of $2^{\circ}$, defined by a circular mask with hard edges.

\section{PSYCHOPHYSICS}

V1 contrast response functions for target Gabors were estimated from contrast discrimination thresholds using a two-interval forced-choice (2IFC) task. For these psychophysical measurements, a Bits ${ }^{++}$digital video processor (Cambridge Research Systems Ltd., UK) was used to provide 14-bit brightness resolution. Eight pedestal contrasts were employed for the target Gabor patches: $0,1,2,4,8,16,32$, and $64 \%$. On each trial, all four targets were presented twice, for $250 \mathrm{~ms}$ in each interval with a $500 \mathrm{~ms}$ blank inter-stimulus interval (ISI). On one of the two intervals in the trial, one of the four targets was incremented in contrast. Subjects maintained fixation on a white square at the center of the stimulus set while indicating the interval ( 1 or 2 ) in which one of the target Gabors increased in contrast; feedback for the task was given by a green ("correct") or red ("incorrect") color at fixation after each response. A 3-down 1-up staircase was used to control the contrast increment on each trial by increasing the contrast increment after trials on which observers provided incorrect answers and decreasing the contrast increment after three correct answers in a row. This staircase converged at a performance level of $79 \%$ correct; the geometric mean of the last three staircase reversals was used as the threshold estimate for each pedestal contrast for each stimulus condition. Several threshold estimates (3-5 runs of 40 trials) at each of the eight contrast levels were completed per subject and averaged.

Threshold versus contrast ( TvC) curves for each subject were fit (using Matlab's lsqcurvefit function) with the derivative of the Naka-Rushton formula (Eq. 1; Boynton et al., 1999),

$R(C)=a C^{p} /\left(C^{p-q}+\sigma^{p-q}\right)$

where $R$ is the estimated neural response (an aggregate measure of input activity and local neural computation), $C$ is the stimulus contrast, $a$ is a scaling constant, and $\sigma, p$, and $q$ are parameters that control the shape of the function. When $C>\sigma$, the function behaves as a simple saturating power law with exponent $q$; when $C<<\sigma$, the neural response is an expansive function with exponent $p-q$. Thus this function describes strong response gain for lowcontrast stimuli and saturation at high contrasts. Parameters used to fit the data for each subject are shown in Table 1.

\section{MRI SYSTEM}

The 7 T magnet (Magnex Scientific, UK) was equipped with a Siemens console (Erlangen, Germany) and a Siemens Avanto head gradient set capable of $80 \mathrm{mT} / \mathrm{m}$ and a maximum slew rate of $333 \mathrm{~T} / \mathrm{m} / \mathrm{s}$. A half volume coil was used for RF transmission and a small $(6 \mathrm{~cm})$ quadrature coil was used for reception (Adriany et al., 2001).

\section{FMRI EXPERIMENTS \\ Main experiment: event-related GE and SE BOLD responses at $7 T$ to isolated Gabor patches}

A single scanning session contained four types of scans: GE BOLD block-design localizers, SE BOLD block-design localizers, GE BOLD event-related runs, and SE BOLD event-related runs. Subjects were engaged in a 2IFC contrast-discrimination task during both localizer and event-related scans; behavioral responses were collected using a fiber-optic button box (Current Designs, Philadelphia, PA, USA). 
Table 1 | Fit parameters for threshold versus contrast functions for the six subjects who responded reliably to the low-contrast stimuli during MR scanning sessions.

\begin{tabular}{llrllr}
\hline Subject & Plot symbol & $\boldsymbol{a}$ & $\boldsymbol{p}$ & $\boldsymbol{q}$ & $\boldsymbol{\sigma}$ \\
\hline 1 & $\diamond$ & 350 & 1.24 & 0.28 & 6.45 \\
$2^{+}$ & $\square$ & 101 & 2.08 & 0.57 & 0.54 \\
$3^{+}$ & $\nabla$ & 143 & 1.77 & 0.44 & 1.12 \\
$4^{+}$ & 0 & 110 & 2.15 & 0.50 & 0.67 \\
$5^{++}$ & $\triangleright$ & 57 & 2.03 & 0.62 & 0.37 \\
6 & $\triangle$ & 349 & 1.17 & 0.29 & 10.54 \\
\hline
\end{tabular}

${ }^{\dagger}$ Excluded from main experiment due to low ROI SNR.

+ Participated in control experiment.

Regions of interest (ROIs) were defined by block-design localizer scans (Figure 1A). "On" and "off" blocks each lasted $12 \mathrm{~s}$; 11 "on" blocks alternated with 10 "off" blocks during each scan, and the first half-cycle ("on") block was discarded before analysis, resulting in a block-alternation frequency of 10 cycles per scan or $1 / 24 \mathrm{~Hz}$. During each block, a new 2IFC trial occurred every $1.5 \mathrm{~s}$ (eight trials per block). During "on" blocks the pedestal contrast for the 2IFC task was $80 \%$; during "off" blocks the target pedestal contrast was $0 \%$. Stimulus durations and ISIs/inter-trial intervals (ITIs) for the task were shorter during these localizers than during the psychophysical and event-related measurements: stimulus duration was $150 \mathrm{~ms}$ and stimuli were separated by a 100-ms ISI. Two or three GE localizer scans and two or three SE localizer scans were included in each experiment, depending on the stamina of the subject.

The event-related scans (Figure 1B) measured BOLD response to four stimulus conditions (target contrasts): 5, 10, 30, and 90\% Michelson contrast (Peli, 1990). Stimuli were presented in the same 2IFC contrast-discrimination task as in the psychophysics $(250 \mathrm{~ms}$ stimulus duration; $200 \mathrm{~ms}$ ISI), with an ITI of 3, 4.5, or $6 \mathrm{~s}$ (ITI was randomly selected and uniformly distributed). There were a total of 48 trials per scan, 12 at each contrast level, for a total of 60-96 presentations of each contrast level for each subject for each BOLD technique (five to eight GE BOLD event-related scans and five to eight SE BOLD event-related scans per subject). Because there were only 12 trials per scan, behavioral data from the scanning sessions were not useful for verifying psychophysical contrast discrimination thresholds, but the response percentage was used to exclude subjects from further analysis - two subjects failed to respond to a majority of the $5 \%$ contrast stimulus presentations, so only data from 6 subjects were analyzed. (Data from the sixth subject were discarded due to low signal-to-noise ratio (SNR), as described below, for a total of five subjects in the main experiment.)

EPI data were acquired with a field of view of $128 \mathrm{~mm} \times 96 \mathrm{~mm}$ and a matrix size of $64 \times 48(6 / 8$ partial Fourier) for a nominal in-plane resolution of $2 \mathrm{~mm}$ isotropic. Slice thickness was $2 \mathrm{~mm}$, volume repetition time (TR) was $1.5 \mathrm{~s}$, and 12 slices were prescribed perpendicular to the calcarine sulcus in an oblique coronal orientation to cover primary visual cortex. For the GE BOLD data echospacing was $0.41 \mathrm{~ms}$ [total slice read-out time $\left(T_{\mathrm{RO}}\right): 14.8 \mathrm{~ms}$ ], echo time (TE) was $20 \mathrm{~ms}$, flip angle $(\alpha)$ was $65^{\circ}$; for the SE BOLD data echo-spacing was $0.41 \mathrm{~ms}\left(T_{\mathrm{RO}}=14.8 \mathrm{~ms}\right)$, TE was $50 \mathrm{~ms}$, $\alpha$ was $90^{\circ}$.

\section{Control experiment: event-related GE and SE BOLD responses at $7 T$ to} large sinusoidal grating patches

The experiment design and data acquisition were identical to the main experiment except the Gabor patches were replaced by larger patches of sinusoidal gratings subtending $4^{\circ}$ of visual angle. Four of the subjects who participated in the main experiment participated in the control.

\section{FMRI DATA ANALYSIS: PREPROCESSING AND LOCALIZERS}

Preprocessing of the functional data, which included motion compensation, high-pass filtering (removal of temporal frequencies below four cycles per scan for localizers, eight cycles per scan for event-related scans) and alignment of functional data to the reference anatomy (Nestares and Heeger, 2000) was accomplished with custom Matlab code. GE EPI and SE EPI functional runs were acquired in an interleaved manner throughout the scanning session and were co-registered via motion compensation. After motion compensation, but before high-pass filtering, fieldmap-based distortion compensation for the EPI images was completed with FSL (Smith et al., 2004).

Prior to participating in the fMRI experiments each subject participated in a separate retinotopic mapping session, which included acquisition of an MP-RAGE anatomy (1 $\mathrm{mm}$ isotropic resolution) for anatomical reference and cortical surface definition. Gray/white matter segmentation, cortical surface reconstruction, and surface inflation and flattening were completed in SurfRelax (Larsson, 2001). Standard retinotopic mapping using rotating wedges and expanding rings (Sereno et al., 1995; DeYoe et al., 1996; Engel et al., 1997) was used to identify V1 and an iso-eccentricity band centered at $3^{\circ}$ of visual angle from the fovea. Boundaries for visual areas were translated to the reference anatomy, and from there to the functional data, to restrict where ROIs would be defined for further analysis.

For each subject, ROIs were selected based on retinotopic location and functional localizers. Repetitions of the GE blockdesign localizers (described above) were averaged together to define GE ROIs, and repetitions of SE localizers were averaged to define SE ROIs. Voxels with coherence (unsigned correlation with a sinusoid at the block-alternation frequency; Bandettini et al., 1993; Engel et al., 1997) exceeding 0.30 in the averaged localizer scans were selected to create the ROIs. This threshold value $(\mathrm{coh}>0.30)$ would correspond to a significance threshold of $p<2 \times 10^{-4}$, uncorrected, if noise in the BOLD data were uncorrelated. Permutation analysis on this particular dataset indicates that this coherence threshold was, on average, associated with a median (across subjects) single-voxel uncorrected significance of $p<4 \times 10^{-4}$ for both the GE BOLD and the SE BOLD data. ROIs were initially defined on a flattened cortical representation, where V1 and eccentricity boundaries could be used to identify the appropriate clusters of voxels. Selected voxels were translated to the in-plane anatomy for further refinement to identify four clusters of contiguous voxels corresponding to the four stimuli in the four visual quadrants. In one subject only the two stimulus representations in the lower visual field (upper bank of the calcarine sulcus) could be unambiguously located in V1, so a total of 22 sub-ROIs in six subjects were identified. 
In the identified ROIs, SNR and contrast-to-noise ratio (CNR) were quantified for both imaging modalities. SNR was characterized as the ratio of the standard deviation (through time) to the mean signal intensity in the event-related scans (i.e., normalized RMS fluctuation). The sub-ROIs for one of the six subjects had high RMS values, compared to the distribution of all 22 (GE RMS greater than 0.02; SE RMS greater than 0.04). In addition, the fit residuals were significantly higher for this subject than for the remaining five subjects when estimating the amplitude of the hemodynamic response (see below), so this subject was excluded from further analysis. Therefore, only 18 sub-ROIs are shown in Figure 2 and used in subsequent analyses. Figure 2A (top) shows the average normalized RMS for individual voxels in each of the 36 identified sub-ROIs (18 SE ROIs and 18 GE ROIs), which is relevant for our sensitivity in defining ROIs. Figure 2A (bottom) shows the normalized RMS calculated for each of the 36 ROIs after averaging all voxels in an ROI; this metric is relevant for our sensitivity in estimating hemodynamic response functions (HRFs) for an ROI. For individual voxels, the average GE SNR is better than the SE SNR (normalized RMS is lower for GE data, and when GE RMS is plotted against SE RMS, most points lie above a line marking a 1:1 ratio). However, for ROIs, the GE and SE SNR are comparable. This is the expected result if greater spatial correlations exist in the noise in GE BOLD because the signal source is dominated by large veins (Yacoub et al., 2005).

Contrast-to-noise ratio was calculated as the ratio of the amplitude of the Fourier component at the block-alternation frequency in the localizer scans ( $80 \%$ stimulus contrast, $0.042 \mathrm{~Hz}$ blockalternation frequency) to the mean amplitude of Fourier components from 60 to 80 cycles per scan $(0.24-0.32 \mathrm{~Hz})$. (Multiple localizer scans were run for each subject, but just one SE localizer and one GE localizer were selected at random from each scanning session for the CNR calculation.) Using the high-frequency end of the Fourier spectrum as a noise baseline avoids contaminating the CNR estimate by low-frequency fluctuations due to residual, uncorrected motion, among other things. In spite of the fact that tissue SNR was comparable in the average signal from SE and GE ROIs (Figure 2A, bottom), the CNR was greater in the GE data (Figure 2B). This result is expected because of the smaller $B O L D$ response surrounding the smaller veins that dominate the SE BOLD signal. CNR is shown both for individual voxels (Figure 2B, top) and for the average signal in each ROI (Figure 2B, bottom). The most informative comparison is between the GE and SE CNR values in the voxels selected by both localizers: $0.34 \pm 0.024$ (cyan points, Figure 2B); Table 2 provides ratios calculated for the mean CNR in other subsets of the voxels in the ROIs. As in the SNR comparison, averaging the signal in the ROI before calculating CNR benefits the SE data more than the GE data (Figure 2B, bottom): if signals from individual voxels are averaged before calculating the CNR, then the ratio of SE CNR to GE CNR (in the SE ROIs) is $0.35 \pm 0.026$.

Coherence values for SE ROIs and GE ROIs are plotted in Figure 2C. Ratios for coherence - which compares the modulation at the stimulus-related Fourier component against the entire Fourier amplitude spectrum, therefore accounting for lowfrequency noise - are also provided in Table 2 and show similar trends, although the SE:GE ratios are consistently higher, presumably because the GE BOLD is more sensitive to physiological noise present in the low-frequency portion of the Fourier spectrum.

For comparison of identified ROI volumes against the expected volume of activation, the size of the cortical territory representing the Gabors was calculated from the average cortical magnification functions in humans reported in Engel et al. (1997), using $2 \sigma$ and $3 \sigma$
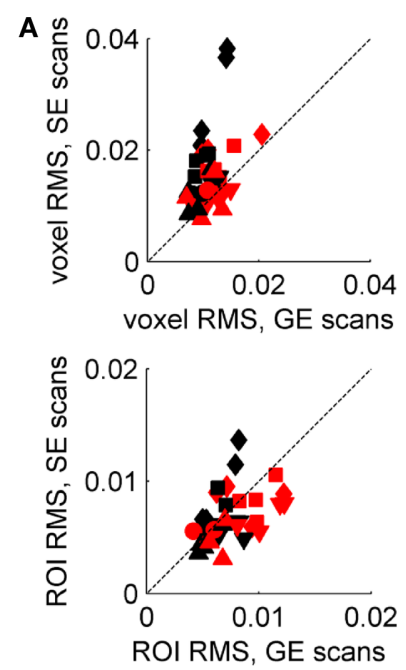
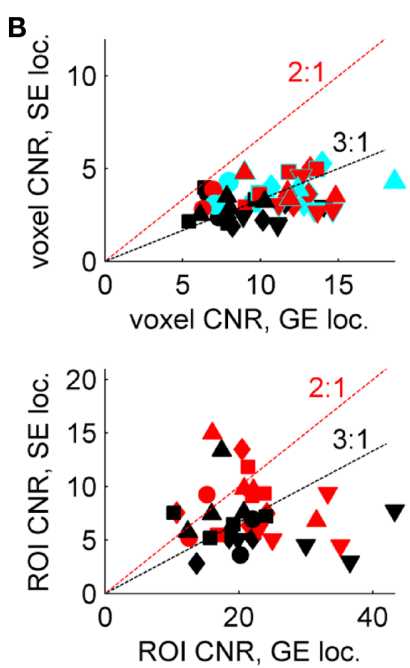
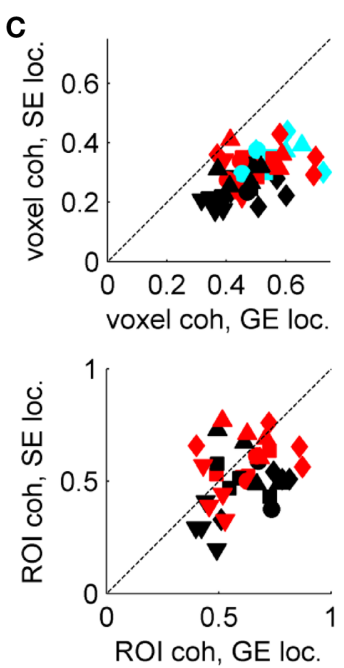

FIGURE 2 | Signal-to-noise ratio and CNR characteristics of SE BOLD and GE BOLD data. (A) Top panel: normalized RMS calculated for a single GE event-related scan (abscissa) and a single SE event-related scan (ordinate), for each voxel in each $\mathrm{ROI}$, then averaged (red symbols: SE ROls; black symbols: GE ROIs; different shapes represent different subjects). Bottom panel: all voxels in each ROI were averaged before calculating RMS. (B) Top panel: average CNR in GE (abscissa) and SE (ordinate) block-design localizer scans for SE ROls (red), GE ROls (black) and voxels included in both types of ROls (cyan). Bottom panel: CNR calculated after averaging all voxels in an ROI. (C) Average voxel coherence (top panel) and $\mathrm{ROI}$ coherence in each type of ROI (bottom panel); colors as in (B). 
Table 2 | Ratios of contrast-to-noise ratio for GE and SE localizers in different voxel populations.

\begin{tabular}{lll}
\hline Voxel population & SE:GE CNR ratio & SE:GE coherence \\
\hline $\begin{array}{l}\text { Voxels in SE ROls } \\
\text { but not GE ROls }\end{array}$ & $0.62 \pm 0.16$ & $0.88 \pm 0.21$ \\
SE ROls & $0.35 \pm 0.026$ & $0.67 \pm 0.038$ \\
$\begin{array}{l}\text { Overlap, SE ROls } \\
\text { and GE ROls }\end{array}$ & $0.34 \pm 0.024$ & $0.65 \pm 0.033$ \\
GE ROls & $0.33 \pm 0.029$ & $0.56 \pm 0.28$ \\
$\begin{array}{l}\text { Voxels in GE ROls } \\
\text { but not SE ROls }\end{array}$ & $0.26 \pm 0.028$ & $0.44 \pm 0.033$ \\
\hline
\end{tabular}

cut-off points to estimate the diameter of the cortical representation of the Gabor patch $\left(0.50^{\circ}\right.$ and $\left.0.75^{\circ}\right)$ and $3 \mathrm{~mm}$ as the estimate of the average local cortical gray matter thickness.

\section{FMRI DATA ANALYSIS: EVENT-RELATED DATA ANALYSIS}

A generalized linear model (GLM) was used to estimate the hemodynamic responses to the stimuli in the event-related design. For the localizer-based analysis, the eight identified sub-ROIs in each subject (four GE and four SE) were first combined to create a single SE ROI and a single GE ROI. Then the data from all voxels in the ROI were averaged before estimating a HRF. For both the localizerbased analysis and the individual-voxel analysis, custom Matlab code estimated the amplitude of the BOLD response (in the average time-course for each ROI or for each voxel) for 12 time points (18 s) after the stimulus onset to avoid making assumptions about the shape of the HRF. Finally, hemodynamic response estimates were fit by a difference-of-gamma (DOG) functions HRF model using the lsqnonlin function in Matlab. The amplitude of the BOLD response was quantified as the peak amplitude of the DOG fit. In a parallel analysis, BOLD response amplitudes were also estimated using the difference between the peak response (4.5-6 s after stimulus onset) and the baseline response (the two HRF points estimated at the time of stimulus presentation); response patterns were identical to the fit amplitude estimates, so only the fit results are shown.

For the voxel-based analysis, individual voxels for which the DOG fit did not meet the following criteria were discarded: timeto-peak amplitude less than $10 \mathrm{~s}$, HRF width less than $6 \mathrm{~s}$, and average error between fit and data less than half the peak amplitude. For each voxel with a successful DOG fit at each contrast, cortical distance from the center of the appropriate sub-ROI was then estimated as the Euclidean distance from the center of the voxel to the center of mass of the sub-ROI. While cortical folding would invalidate this metric for extended ROIs (as in the control experiment), this is a reasonable metric for the small sub-ROIs in the main experiment in which cortical distances were typically less than $5 \mathrm{~mm}$.

\section{RESULTS}

In our first experiment, we measured the GE and SE BOLD contrast response functions for isolated Gabor patches. First, GE BOLD and SE BOLD block-design localizers were used to define two sets of ROIs (Figure 3A). Each set of ROIs in each subject consisted of four sub-ROIs, corresponding to the four cortical representations of the

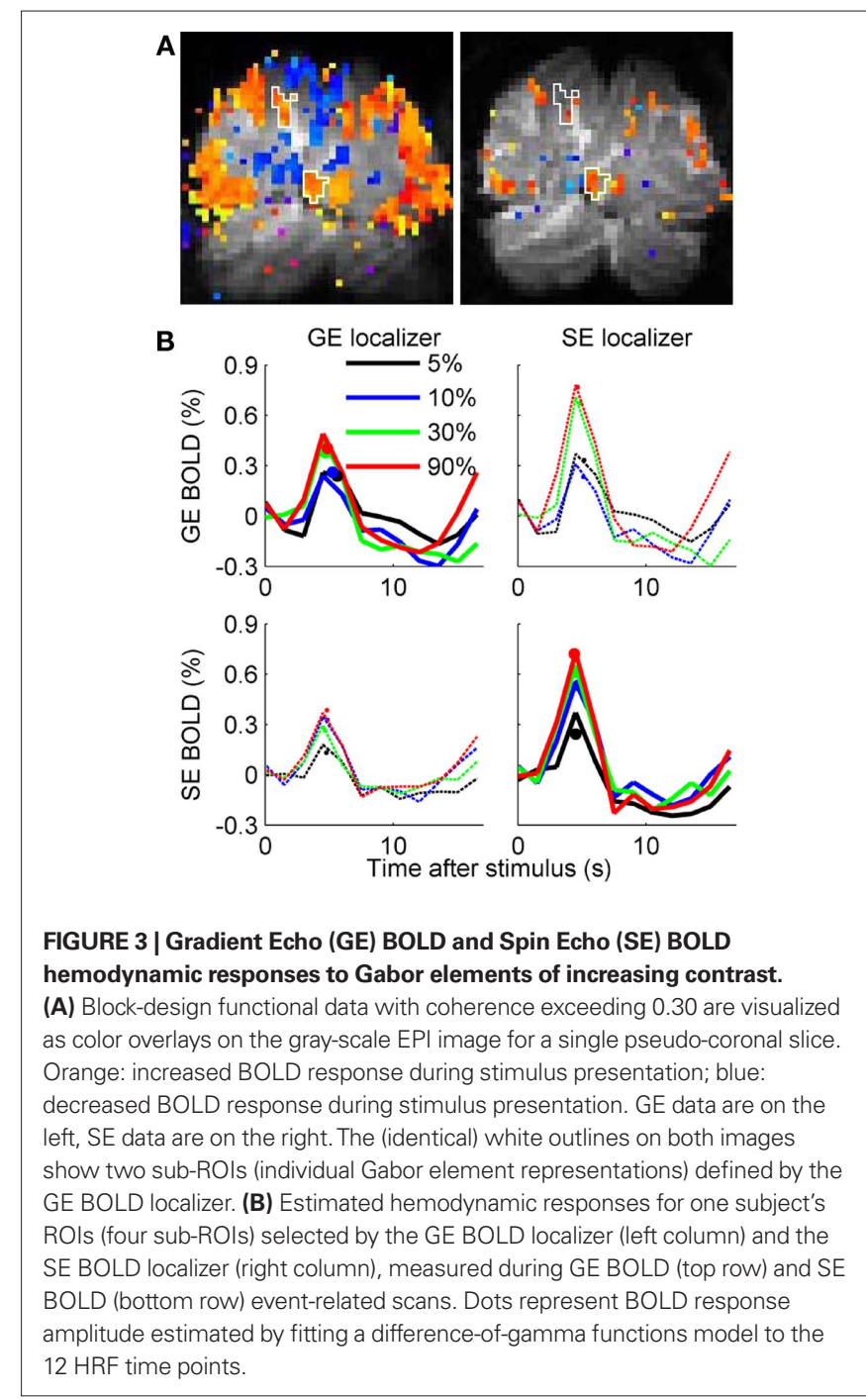

four Gabor patches. The SE BOLD-derived ROIs were smaller than the GE BOLD-derived ROIs, as would be expected either from the lower CNR of the SE BOLD technique (Olman et al., 2010) or from the decreased blurring due to dominance of the small-diameter vascular compartment. The average volume of a SE BOLD subROI was $137 \pm 9.7 \mathrm{~mm}^{3}$ (mean \pm SEM, 18 sub-ROIs); the average volume of a GE BOLD sub-ROI was $288 \pm 22 \mathrm{~mm}^{3}$. The SE ROIs were also largely contained within the larger GE ROIs: $9.2 \pm 2.5 \%$ (mean \pm SEM, 18 sub-ROIs) of the voxels selected by SE localizers were not included in the GE ROIs, while $54 \pm 3.6 \%$ of the voxels in GE ROIs were outside of the smaller SE ROIs. Assuming a cortical thickness of $3 \mathrm{~mm}$, and a cortical representation for each Gabor patch approximately $5-8 \mathrm{~mm}$ in diameter, the predicted stimulated cortical volume in each location is approximately $60-150 \mathrm{~mm}^{3}$.

We then estimated GE BOLD and SE BOLD event-related HRFs within each ROI for Gabor patches presented at 5, 10, 30, and 90\% luminance contrast. Estimated HRFs for the SE and GE BOLD response in the SE and GE BOLD-defined ROIs in one representative subject are shown in Figure 3B. The primary analyses of interest are the GE BOLD response in the GE BOLD-defined ROIs (upper 
right panel) and the SE BOLD response in the SE BOLD-defined ROIs (lower left panel). However, the crossed analyses (GE BOLD response in voxels selected by SE BOLD localizer, and vice versa, thin dashed lines in Figure 3B) were also performed. It is worth noting that both the GE BOLD and the SE BOLD HRFs are larger in the more-selective SE BOLD-derived ROIs (right column).

The average GE and SE BOLD responses to Gabor patches of increasing contrast are shown in Figure 4A. Because the underlying V1 neural response saturates with increasing contrast, a linear increase in amplitude with increasing contrast is not expected. However, in neither the GE nor the SE data was the response to $5 \%$ contrast significantly lower than the response to $10 \%$ contrast. In the SE data, there was a trend in the expected direction $\left(t_{4}=1.74, p=0.08\right.$, one-tailed paired $t$-test $)$, but no distinction in the GE data $\left(t_{4}=0.22, p=0.58\right)$. Normalized BOLD response is plotted against psychophysically estimated neural response for individual subjects in Figure 4B and illustrates the variability within and between subjects that contributed to our failure to measure $5 \%$ contrast responses that were significantly less than $10 \%$ contrast responses.

In a control experiment, we tested whether the lack of reliability in the estimated BOLD response to low contrast targets was a consequence of the small stimulus size or of the rapid eventrelated experiment design. The rapid event-related design (average ISI of $4.5 \mathrm{~s}$ ) assumes linearity (specifically, superposition) of the hemodynamic response; if this assumption is not valid, it could produce unreliable behavior, particularly in the weaker responses. Therefore, with an experiment design and analysis identical to the previous, we measured the BOLD response to larger patches of sinusoidal gratings $\left(2^{\circ}\right.$ radius) centered at the same eccentricity $\left(3^{\circ}\right)$ as the four Gabor patches in the main experiment. Four of the subjects who participated in the main experiment participated in this control. As shown in Figure 4B, the GE BOLD response at $10 \%$ contrast was significantly larger than the response at $5 \%$ $\left(t_{3}=-4.04, p=0.014\right.$, one-tailed paired $t$-test $)$. The SE BOLD response at $10 \%$ contrast was also higher than the response to $5 \%$ contrast $\left(t_{3}=-2.32, p=0.052\right)$.

To investigate the contribution of voxel selection to the unreliable estimates of the BOLD response to isolated Gabor patches, we estimated HRFs for individual voxels in the GE BOLD ROIs from the main experiment (Figure 5). As expected, since the stimulus contrast is highest in the center of the Gabor patch and decreases toward the edges, response magnitude decreased with distance from the center of the cortical representation of each Gabor patch (Figure 5A). What is unexpected, however, is the spatial dependence of the ratio between the 10 and 5\% contrast responses (Figure 5B). In every subject, this ratio was fit by a linear trend with negative slope (GE data: $t_{4}=-4.3, p=0.013$; SE data: $\left.t_{4}=-2.7, p=0.052\right)$. The average intercept was greater than 1 (GE data: $1.24 \pm 0.35$ (SD); SE data: $1.3 \pm 0.27)$, indicating that the $10 \%: 5 \%$ ratio is greater than 1 in the center of the ROI, but decreases with distance from the center of the ROI. Therefore, the exact spatial extent of the ROI can have a strong effect on the estimated amplitude of the response to a $5 \%$ contrast target, relative to a $10 \%$ contrast target, which can explain the variability between subjects observed in the ROI analysis. Using the $y$-intercept of the linear fits illustrated in Figure 5A as an estimate of the amplitude of the BOLD response to the Gabor patch improves the linearity of the contrast response functions recovered for both GE and SE BOLD (Figure 5C): 95\% confidence intervals (shaded regions) are 14\% smaller in the top panel for the GE data, and $18 \%$ smaller in the top panel for the SE data.

\section{DISCUSSION}

Our main finding is that the average response in a selected ROI is not the most reliable method of estimating the BOLD response for isolated stimuli that produce small patches of cortical activation, particularly for GE BOLD. In an ROI-based analysis, we found that the GE BOLD response to small image patches presented at $5 \%$
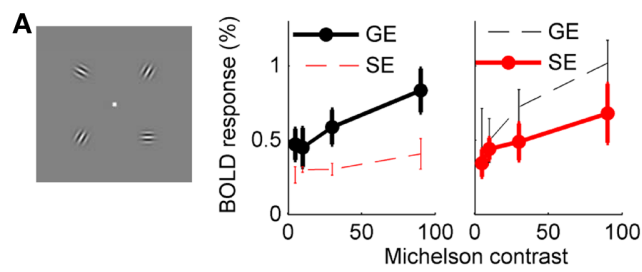

C

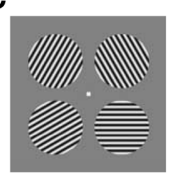

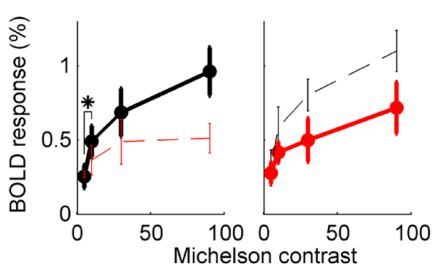

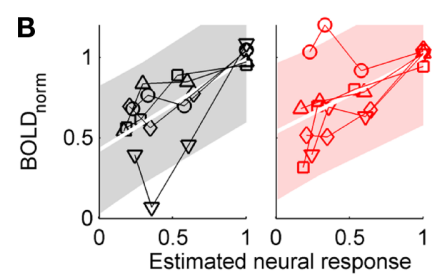

D

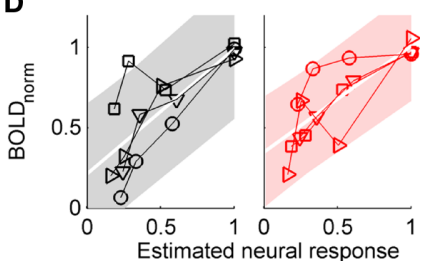

FIGURE 4 | Contrast response functions measured with GE and SE BOLD. (A) Main experiment, using Gabor elements as stimuli. For each type of ROI (GE localizers on top, SE localizers on bottom), the event-related GE and SE BOLD responses to isolated Gabor patches presented at 5, 10, 30, and 90\% contrast were estimated for five subjects. Solid black line indicates GE BOLD CRF in GE BOLD-defined ROI; solid red line indicates SE BOLD CRF in SE BOLD ROI.
Dashed lines indicate crossed analyses (GE BOLD CRF in SE BOLD ROI, and vice versa). Error bars indicate SEM. Asterisk indicates $p<0.05$, paired $t$-test, $n=5$. (B) Normalized BOLD data are plotted against psychophysical estimates of contrast response in individual subjects, GE data in black on left, SE data in red on right. Shaded area indicates 95\% confidence intervals for a linear fit (white line) to the data. (C,D) Control experiment, using grating patches as stimuli $(n=4)$. 

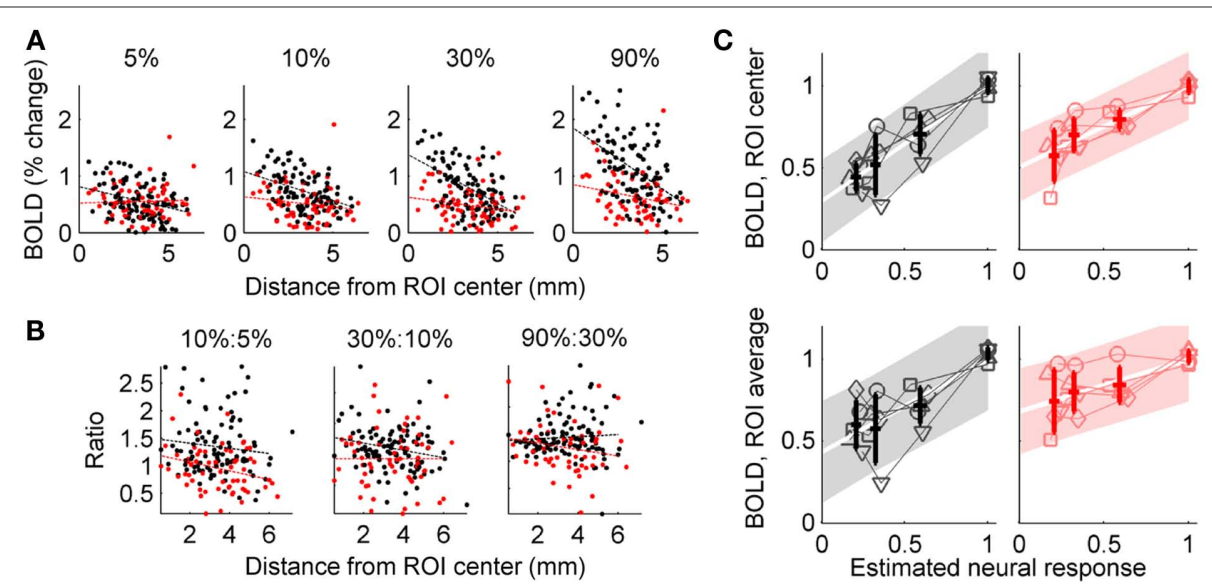

FIGURE 5 | Voxel-based analysis of BOLD contrast response. (A) Response amplitude estimated for individual voxels in the GE BOLD-defined sub-ROIs in a representative subject (data from four sub-ROls combined). Black dots indicate estimated GE BOLD response; red dots indicate estimated SE BOLD response. Dashed lines indicate linear fit to response amplitude as a function of distance from sub-ROI center. (B) Ratios of GE BOLD (black) and SE BOLD (red) responses to neighboring contrasts. Only the trend in the GE BOLD $10 \%: 5 \%$ ratio was significant. (C) Normalized BOLD response in the GE-defined ROI plotted against individual subjects' psychophysically derived estimates of the neural response. Thin lines indicate individual subject data; bold points indicate average BOLD response $( \pm S D)$, with abscissa coordinate determined by average estimated neural response (mean \pm SD). Shaded region indicates $95 \%$ confidence interval for linear regression. Top: BOLD response amplitude estimated from the $y$-intercept of the linear fit to amplitude as a function of distance from the center of the cortical representation of the Gabor patch; bottom: BOLD response estimated as the average of the voxel responses (ignoring spatial location). Left: GE BOLD responses; right; SE BOLD responses. contrast was not significantly smaller than the response to patches presented at $10 \%$ contrast. The difficulty in distinguishing 5 and $10 \%$ contrast responses was not an artifact of the experiment design, because the GE BOLD response to extended sinusoidal grating patches presented at $10 \%$ contrast was significantly larger than the response to $5 \%$ contrast gratings. Nor was it a consequence of low CNR in the data, because subjects with low CNR or high fit residuals were excluded from analysis, and the non-monotonic behavior observed in some subjects was more pronounced in the GE BOLD data, which had higher CNR than the SE BOLD data. The larger-than-expected GE BOLD response in the ROI-based analysis appeared to be a consequence of spatial heterogeneity in the cortical response to small stimuli. Specifically, we observed a relative amplification of the 5\% contrast response at the edges of the cortical representation of the stimuli. Improved contrast response estimates were obtained, particularly for the GE BOLD data, by fitting individual voxels and estimating the magnitude of the BOLD response at the center of the ROI.

Our finding of an amplification of the GE BOLD ROI response at very low contrast is not without precedent. Murray (2008) measured the effect of attention on V1 contrast response functions for relatively small patches of sinusoidal gratings; the main finding was that the effects of attention to patches of sinusoidal gratings presented in the four visual quadrants could be described by a baseline shift. Close inspection of Figure 3 in that paper, however, shows that the GE BOLD response to the $3 \%$ contrast stimulus is larger than the BOLD response to the $6 \%$ contrast grating, most notably in the unattended condition. The stimuli in the Murray experiment subtended $6^{\circ}$ of visual angle, centered at $8^{\circ}$ eccentricity, so the predicted size of the cortical representation of those stimuli should be intermediate between the Gabor patches and the larger sinusoidal gratings used in this experiment.
This is also not the first time we have observed that voxel selection, or spatial heterogeneity in the GE BOLD responses in $\mathrm{V} 1$, can have an impact on interpretations of the relationship between BOLD and stimulus geometry or psychophysical measurements. We have observed previously that some voxels produce negative hemodynamic impulse responses to the presentation of a spatially restricted checkerboard (Olman et al., 2007), even though the stimulus is presented in the voxel's receptive field (i.e., the voxel is inside a ROI defined by a differential localizer that clearly reflects retinotopic location within $\mathrm{V} 1$ ). The local dominance of the negative BOLD in that study was explained by mislocalization of signal by large veins, which brought surrounding negative BOLD signal into voxels in the cortical territory representing the stimulus. More recently, we have shown that surround suppression of the BOLD response to isolated Gabors cannot be predicted from psychophysical measurements of the suppressed visual response to the target Gabors (Schumacher and Olman, 2010). When a target Gabor was flanked by parallel Gabor elements at higher contrast, we measured a localized V1 GE BOLD signal that decreased as the contrast of the target Gabor increased. Those two studies, in combination with the present findings, indicate that accurate inference of spatially heterogeneous neural activity from high-resolution BOLD data requires a model for neurohemodynamic coupling that is more complex than a Gaussian pooling kernel (Engel et al., 1997; Parkes et al., 2005). These experiments also suggest the necessity of a model that takes into account more complex spatiotemporal characteristics of vascular pooling (Kriegeskorte et al., 2009).

One possible neurohemodynamic explanation for the unexpected decrease in the ratio of 10\%:5\% contrast responses with distance from the center of the cortical representation of each patch is a spatial broadening of the cortical perfusion in response to $5 \%$ contrast stimuli. If the hemodynamic response becomes more 
narrowly targeted (better matched to the locus of neural activity) as contrast increases, then voxels at the edge of a generously defined ROI would show more positive BOLD response for 5\% contrast than $10 \%$ contrast stimuli. This explanation for the data is essentially an argument in favor of hemodynamic spatial uncertainty for low-contrast stimuli, i.e., hemodynamic resources that are directed to an overly large region of cortex for small, low-contrast stimuli. The GE BOLD ROIs, sensitive to signals from larger veins that pool over a larger cortical territory, would be particularly sensitive to excessive perfusion of flanking cortical territory.

While spatially untargeted perfusion might explain our pattern of results (i.e., variability between subjects in the relative amplitudes of responses to 5\% contrast and 10\% contrast Gabors in an ROI-based analysis), this is certainly not a general expectation for fMRI experiments. There has been ample demonstration that the arterial blood supply as well as GE BOLD and SE BOLD responses are regulated on a spatial scale fine enough to enable visualization of individual cortical columns - a scale much finer than the resolution required for the present experiment (Cheng et al., 2001; Duong et al., 2001; Yacoub et al., 2008). However, these studies that show excellent targeting of perfusion have been performed with large stimuli using a differential, block-design protocol that minimizes the contribution of large veins and measures only perturbations of the blood supply from a high-response state.

As an alternative to the hemodynamic explanation above, subjects' eye-movements could conceivably explain the relative increase in the strength of the BOLD response to $5 \%$ contrast stimuli in voxels far from the ROI center. If subjects are more likely to make micro-saccades in the direction of one of the target stimuli when the stimuli are near detection threshold (and therefore harder to see), then the cortical representation for the 5\% contrast stimuli would be blurred or expanded. Thus, in voxels several millimeters from the ROI center, where the BOLD responses to high-contrast stimuli (with more stable fixation) are decreasing, the response to $5 \%$ contrast stimuli (spread by fixation instability) would be inordinately large. Eye-tracking equipment is not currently available at our $7 \mathrm{~T}$ scanner, so we do not have data regarding fixation stability on each trial. In addition to repeating this experiment with eye-tracking, another way to distinguish the hyperperfusion hypothesis from the micro-saccade hypothesis is perfusion measurements. With independent measurement of BOLD response and perfusion responses, we could determine whether the balance of perfusion and oxygen consumption differs across the cortical territory for near-threshold and supra-threshold stimuli.

Finally, as another alternative explanation for our data, it is possible that the amplification of the observed BOLD response to 5\% contrast is driven by attention or anticipation. Robust V1 BOLD responses occasionally represent perception, rather than actual stimulus presence or contrast (Ress and Heeger, 2003; Maier et al., 2008). A recent optical imaging study also found that anticipation of a stimulus produced a robust hemodynamic response that was not accompanied by measured neural responses (Sirotin and Das, 2009). Since response rates for $5 \%$ contrast Gabors were lower than for the higher contrast patterns (80-85\% instead of 95-100\%), perhaps the sheer difficulty of reliably perceiving 5\% contrast Gabors at $3^{\circ}$ eccentricity resulted in a unique neural modulation akin to modulation by attention (Buracas and Boynton, 2007; Murray,
2008). Therefore modulation by attention, in spite of the assigned contrast discrimination task, could disproportionately amplify the neural (and BOLD) response at 5\% contrast under the particular conditions of the experiment we conducted. However, there are several reasons that such cognitive differences are not likely to explain the observed pattern. First, it would be difficult to explain why an attention or anticipation effect would be observed more strongly in the GE BOLD signal than in the SE BOLD signal. Using attention to explain the full pattern of results we observed would require invoking an attention-based non-linearity in the pooling of blood from small-diameter venous compartments to largediameter venous compartments, which returns us to an argument similar to the hyperperfusion argument presented above. Second, Murray (2008) found an amplification of the BOLD response to 3\% contrast stimuli in both the unattended and attended conditions, further suggesting that mechanisms related to perceptual awareness are not responsible for the pattern observed. Finally, our ITI was randomized, so subjects could not anticipate the onset timing for low-contrast trials.

For comparing the BOLD data against the underlying neural response in this study, we used psychophysical data to estimate neural contrast response functions in V1. While the use of contrast discrimination threshold measurements to estimate V1 contrast response functions has been validated in the past (Boynton et al., 1999; Zenger-Landolt and Heeger, 2003), it is nonetheless an indirect estimate of the neural population response. Electrophysiological data from non-human primates suggests that the population contrast response function can be fit by a power law with exponent $\sim 0.6$ (Heeger et al., 2000); this power law predicts a ratio of 1.5 between the magnitude of the neural population responses to 10 and $5 \%$ contrast gratings. The psychophysical data predict an average ratio of 1.6. Both ratios are more consistent with the BOLD response estimated by the extrapolated intercept at the center of the cortical representations of the patches [voxel-based analysis, GE data: $1.24 \pm 0.35$ (standard deviation); SE data: $1.3 \pm 0.27$ ] than with the ROI-based analysis of the GE data $(0.95 \pm 0.50$, Figure $4 A)$. Presumably due to the smaller ROI size and reduced contribution of large vein data to the SE signal, the SE ROI-based analysis provided more reliable estimates of the underlying neural responses (than the GE ROIbased analysis) and was most consistent with the psychophysical data and the voxel-based analysis $(1.43 \pm 0.52)$.

The ROI-based analysis provided a more reliable estimate of the underlying neural response to isolated Gabor patches when applied to the SE BOLD data than when applied to the GE BOLD data. In part this was because the SE-defined ROIs were more spatially restricted (roughly half the volume of the GE-defined ROIs), and in part this was because the spatial heterogeneity was not as pronounced (the negative trend for the 10\%:5\% response ratio was present but not significant across subjects). All of the potential explanations presented above for the overly large response to $5 \%$ contrast stimuli we observed at the periphery of the sub-ROIs should affect SE data as well as GE data. However, because the GE data contain a stronger signal contribution from large veins, which pool signal over a larger cortical territory, it is likely that this spatial filtering (blurring) in the GE data amplifies local variations in the BOLD response. 
While the main experiment suggests that smaller ROIs (limited to just the center of the stimulated cortical region) would provide better estimates of the BOLD response, using small ROIs is problematic because they are difficult to localize accurately and because ROIs that include just a few voxels are generally quite noisy. The approach used in this paper - to define a generous ROI and then fit the individual voxel responses to estimate the response at the center - addresses both the problem of localization and the need to combine data from a large number of voxels to beat down noise. This approach is only relevant for ROIs in which the BOLD response is expected to vary significantly throughout the ROI. With large stimuli, only a small percentage of the voxels selected for analysis might be on the edge (i.e., in cortical territory where the neural response magnitude varies strongly as a function of spatial position), but with small stimuli, the neural response magnitude varies consistently throughout even a conservatively defined ROI.

To summarize, the GE BOLD response to parafoveal Gabor elements presented at 5\% contrast is not reliably smaller than the response to $10 \%$ contrast elements in an analysis based on the average response in an ROI. The ratio of 10\%:5\% responses decreases with cortical distance from the center of the ROI - exactly opposite the pattern predicted if the size of the cortical representation of a Gabor patch increases with contrast. Either eye movements or poor spatial targeting of perfusion for weak stimuli (vascular spatial uncertainty) could explain this result, which was more pronounced for GE BOLD than for SE BOLD data. While it appears likely that the limitations observed in the GE BOLD ROI-based analysis would be encountered only rarely - response estimation was more reliable with higher contrast stimuli and when stimuli are increased to subtend several degrees of visual angle - this study offers an important cautionary note for the interpretation of high-resolution GE BOLD measurements of the cortical representation of finely detailed visual stimuli.

\section{ACKNOWLEDGMENTS}

The authors would like to thank S. O. Murray for helpful discussions regarding this project, as well as the following funding sources: NIH-NPCS Graduate Student Training Fellowship, the MIND Foundation, the Keck Institute, and the following NIH grants: BTRR P41 RR008079, P30 NS057091, R01 EY015261.

\section{REFERENCES}

Adriany, G., Pfeuffer, J., Yacoub, E., van de Moortele, P. F., Shmuel, A., Andersen, P., Hu, X., Vaughan, J. T., and Ugurbil, K. (2001). "A half-volume transmit/ receive coil combination for 7 Tesla applications," in Paper Presented at the 9th Annual Scientific Meeting of the ISMRM, Glasgow.

Bandettini, P. A., Jesmanowicz, A., Wong, E. C., and Hyde, J. S. (1993). Processing strategies for time-course data sets in functional MRI of the human brain. Magn. Reson. Med. 30, 161-173.

Bandettini,P.A., Wong, E.C., Jesmanowicz, A., Hinks, R. S., and Hyde, J. S. (1994). Spin-echo and gradient-echo EPI of human brain activation using BOLD contrast: a comparative study at $1.5 \mathrm{~T}$. NMR Biomed. 7, 12-20.

Boynton, G. M., Demb, J. B., Glover, G. H., and Heeger, D. J. (1999). Neuronal basis of contrast discrimination. Vision Res. 39, 257-269.

Boynton, G. M., Engel, S. A., Glover, G. H., and Heeger, D. J. (1996). Linear systems analysis of functional magnetic resonance imaging in human V1. J. Neurosci. 16, 4207-4221.

Brainard, D.H. (1997). The Psychophysics Toolbox. Spat. Vision 433-436.

Buracas, G. T., and Boynton, G. M. (2007). The effect of spatial attention on contrast response functions in human visual cortex. J. Neurosci. 27, 93-97.

Cheng, K., Wagooner, R. A., and Tanaka, K. (2001). Human ocular dominance columns as revealed by high-field functional magnetic resonance imaging. Neuron 32, 359-374.

DeYoe, E. A., Carman, G. J., Bandettini, P. A., Glickman, S., Wieser, J., Cox, R.
W., Miller, D., and Neitz, J. (1996). Mapping striate and extrastriate visual areas in human cerebral cortex. Proc. Natl. Acad. Sci. U.S.A. 93, 2382-2386.

Duong, T. Q., Kim, D.-S., Ugurbil, K., and Kim, S.-G. (2001). Localized cerebral blood flow response at sub-millimeter columnar resolution. Proc. Natl. Acad. Sci. U.S.A. 95, 11489-11492.

Duong, T. Q., Yacoub, E., Adriany, G., Hu, X., Ugurbil, K., and Kim, S.-G. (2003). Microvascular BOLD contribution at 4 and $7 \mathrm{~T}$ in the human brain: gradient-echo and spin-echo fMRI with suppression of blood effects. Magn. Reson. Med. 49, 1019-1027.

Engel, S. A., Glover, G. H., and Wandell, B.A. (1997). Retinotopic organization in human visual cortex and the spatial precision of functional MRI. Cereb. Cortex 7, 181-192.

Heckman, G. M., Bouvier, S. E., Carr, V. A., Harley, E. M., Cardinal, K. S., and Engel, S. A. (2007). Nonlinearities in rapid event-related fMRI explained by stimulus scaling. Neuroimage 34, 651-660.

Heeger, D. J., Huk, A. C., Geisler, W. S., and Albrecht, D. G. (2000). Spikes versus BOLD: what does neuroimaging tell us about neuronal activity? Nat. Neurosci. 3, 631-633.

Hulvershorn, J., Bloy, L., Gualtieri, E. E., Leigh, J. S., and Elliott, M. A. (2005). Spatial sensitivity and temporal response of spin echo and gradient echo bold contrast at $3 \mathrm{~T}$ using peak hemodynamic activation time. Neuroimage 24, 216-223.

Kriegeskorte, N., Cusack, R., and Bandettini, P. A. (2009). How does an $\mathrm{fMRI}$ voxel sample the neuronal activity pattern: compact-kernel or complex-spatiotemporal filter? Neuroimage 49, 1965-1976.

Larsson, J. (2001). Imaging Vision: Functional Mapping of Intermediate Visual Processes in Man. Unpublished $\mathrm{PhD}$ thesis, Karolinska Institutet, Stockholm.

Logothetis, N. K., Pauls, J., Augath, M., Trinath, T., and Oeltermann, A. (2001). Neurophysiological investigation of the basis of the fMRI signal. Nature 412, 150-157.

Maier, A., Wilke, M., Aura, C., Zhu, C., Ye, F. Q., and Leopold, D. A. (2008). Divergence of $\mathrm{fMRI}$ and neural signals in V1 during perceptual suppression in the awake monkey. Nat. Neurosci. 11, 1193-1200.

Murray, S. O. (2008). The effects of spatial attention in early visual cortex are stimulus independent. J. Vision 8, 1-11.

Nestares, O., and Heeger, D. J. (2000). Robust multiresolution alignment of MRI brain volumes. Magn. Reson. Med. 43, 705-715.

Norris, D. G., Zysset, S., Mildner, T., and Wiggins, C. J. (2002). An investigation of the value of spin-echo-based fMRI using a Stroop color-word matching task and EPI at 3 T. Neuroimage 15, 719-726.

Ogawa, S., Menon, R. S., Tank, D. W., Kim, S.-G., Merkle, H., Ellerman, J. M., and Ugurbil, K. (1993). Functional brain mapping by blood oxygenation level-dependent contrast magnetic resonance imaging. A comparison of signal characteristics with a biophysical model. Biophys. J. 64, 803-912.
Oja, J. M. E., Gillen, J., Kauppinen, R. A., Kraut, M., and van Zijl, P.C.M. (1999). Venous blood effects in spin-echo fMRI of human brain. Magn. Reson. Med. 42, 617-626.

Olman, C. A., Inati, S., and Heeger, D. J. (2007). The effect of large veins on spatial localization with GE BOLD at $3 \mathrm{~T}$ : displacement, not blurring. Neuroimage 34, 1126-1135.

Olman, C. A., Van de Moortele, P.-F., Schumacher, J. F., Guy, J., Ugurbil, K., and Yacoub, E. (2010). Retinotopic mapping with Spin Echo BOLD at 7 Tesla. Magn. Reson. Imaging 28, 1258-1269.

Parkes, L. M., Schwarzbach, J. V., Bouts, A. A., Deckers, R. H. R., Pullens, P., Kerskens, C. M., and Norris, D. G. (2005). Quantifying the spatial resolution of the gradient echo and spin echo BOLD response at 3 Tesla. Magn. Reson. Med. 54, 1465-1472.

Peli, E. (1990). Contrast in complex images.J.Opt. Soc. Am. A 7, 2032-2040

Pelli, D. G. (1997). The VideoToolbox software for visual psychophysics: transforming numbers into movies. Spat. Vision 10, 437-442.

Ress, D., and Heeger, D. J. (2003). Neuronal correlates of perception in early visual cortex. Nat. Neurosci. 5, 414-420.

Schumacher, J. F., and Olman, C. A. (2010). High-resolution BOLD fMRI measurements of local orientationdependent contextual modulation show a mismatch between predicted V1 output and local BOLD response. Vision Res. 50, 1214-1224.

Sereno, M. I., Dale, A. M., Repas, J. B., Kwong, K. K., Belliveau, J. W., Brady, T. J., Rosen, B. R., and Tootell, R. B. 
(1995). Borders of multiple visual areas in humans revealed by functional magnetic resonance imaging. Science 268, 889-893.

Sirotin, Y. B., and Das, A. (2009). Anticipatory haemodynamic signals in sensory cortex not predicted by local neuronal activity. Nature 457, 475-479.

Smith, S. M., Jenkinson, M., Woolrich, M. W., Beckmann, C. F., Behrens, T. E. J., Johansen-Berg, H., Bannister, P. R., De Luca, M., Drobnjak, I., Flitney, D. E., Niazy, R. K., Saunders, J., Vickers, J., Zhang, Y., De Stefano, N., Brady, J. M. and Matthews, P.M. (2004). Advances in functional and structural MR image analysis and implementation as FSL. Neuroimage 23(Suppl. 1), 208-219.

Yacoub, E., Duong, T. Q., Van de Moortele, P.-F., Lindquist, M., Adriany, G., Kim, S.-G., Ugurbil, K., and Hu, X. (2003). Spin-echo fMRI in humans using high spatial resolutions and high magnetic fields. Magn. Reson. Med. 49, 655-664.

Yacoub, E., Harel, N., and Ugurbil, K. (2008). High-field fMRI unveils orientation columns in humans. Proc. Natl. Acad. Sci. U.S.A. 105, 10607-10612.

Yacoub, E., Van de Moortele, P.F., Shmuel, A., and Ugurbil, K. (2005). Signal and noise characteristics of Hahn SE and GE BOLD fMRI at $7 \mathrm{~T}$ in humans. Neuroimage 24, 738-750.
Zenger-Landolt, B., and Heeger, D. J. (2003). Response suppression in V1 agrees with psychophysics of surround masking. J. Neurosci. 23, 6884-6893.

Zhang, N., Zhu, X.-H., and Chen, W. (2008). Investigating the source of BOLD nonlinearity in human visual cortex in response to paired visual stimuli. Neuroimage 43, 204-212.

Conflict of Interest Statement: The authors declare that the research was conducted in the absence of any commercial or financial relationships that could be construed as a potential conflict of interest.
Received: 31 August 2010; accepted: 10 March 2011; published online: 24 March 2011.

Citation: Schumacher JF, Thompson SK and Olman CA (2011) Contrast response functions for single Gabor patches: ROIbased analysis over-represents low-contrast patches for GE BOLD. Front. Syst. Neurosci. 5:19. doi: 10.3389/fnsys.2011.00019 Copyright $@ 2011$ Schumacher, Thompson and Olman. This is an open-access article subject to a non-exclusive license between the authors and Frontiers Media SA, which permits use, distribution and reproduction in other forums, provided the original authors and source are credited and other Frontiers conditions are complied with. 